\title{
Impacto de la precipitación pluvial en el agua renovable como análisis de su aprovechamiento en México
}

\author{
Impact of rainfall on renewable water as an analysis of its use in Mexico \\ Impacto da precipitação pluvial sobre as águas renováveis como uma \\ análise de seu uso no México
}

Daniela I. Castillejos Moguel

Arquitecta, M.Sc. Estudios Sustentables, Regionales y Metropolitanos

Universidad Autónoma del Estado de México, México.

danytsa_arqui@hotmail.com

(iD) https://orcid.org/0000-0002-2784-9544

Recibido: junio 15 de 2021

Aceptado: noviembre 19 de 2021

Publicado: noviembre 25 de 2021

\section{RESUMEN}

El propósito del presente artículo es analizar uno de los elementos vitales para el ser humano: el agua. Al hacerlo - a través de algunas de sus manifestaciones y comprender sus principales conceptos-, surge el cuestionamiento referente al agua pluvial y su aprovechamiento. A partir de esto se realiza una prueba estadística con la cual se comprueba la hipótesis de que, en efecto, sí existe una relación entre el agua pluvial y el agua renovable. En consecuencia, surgen cuestionamientos de la razón de la escasez de agua en el país, así como de la explotación del mencionado recurso obteniendo diversas conclusiones.

Palabras clave: Precipitación; agua; aprovechamiento de recursos.

\begin{abstract}
The purpose of this article is to analyze one of the vital elements for human beings: water. In doing so -through some of its manifestations and understanding its main concepts - the question arises regarding rainwater and its use. Based on this, a statistical test is carried out with which the hypothesis is verified that, in effect, there is a relationship between rainwater and renewable water. Consequently, questions arise about the reason for the scarcity of water in the country, as well as the exploitation of this resource, obtaining various conclusions.
\end{abstract}

Keywords: Precipitation; water; use of resources. 


\section{RESUMO}

O objetivo deste artigo é analisar um dos elementos vitais para o ser humano: a água. Ao fazê-lo - por meio de algumas de suas manifestações e compreensão de seus principais conceitos- surge a questão a respeito da água da chuva e seu uso. Com base nisso, é realizado um teste estatístico com o qual se verifica a hipótese de que, de fato, existe uma relação entre a água da chuva e a água renovável. Consequentemente, surgem questionamentos sobre o motivo da escassez de água no país, bem como a exploração deste recurso, obtendose várias conclusões.

Palavras-chave: Precipitação; Água; uso de recursos.

\section{INTRODUCCIÓN}

Acerca de México, se puede afirmar que existe un gran cambio a nivel territorial, ambiental y social derivado de las trasformaciones económicas que se han presentado a lo largo de los años, como la industrialización. Hoy en día, los cambios que presentan, principalmente las ciudades, han provocado que se necesiten cada día más recursos naturales ya sea para necesidades básicas o bien para abastecer actividades económicas. Desgraciadamente, son los factores económicos y sociales los que están acabando con estos recursos naturales contaminándolos y extinguiéndolos (Gudynas, 2001).

Como resultado, surge una problemática donde el peligro de las posibilidades de continuar utilizando los recursos naturales es cada vez mayor. Esto se deriva de la destrucción de las fuentes de materia prima así como la explotación de recursos y la contaminación de estos, rebasando su capacidad de renovación (Rodríguez, 2014).

Por otro lado, la segregación de regiones vista como consecuencia de dichas problemáticas, divide territorios para modificarlos y crear ciudades, lo que ocasiona que dichas regiones, las cuales aún no se adaptan a los cambios actuales, queden desprotegidas social, ambiental y políticamente (Boisier, 2001). Esto implica que los organismos públicos pongan mayor atención en las regiones globalizadas en cuestión de gestión y distribución de recursos, por ejemplo el agua.
Por lo que refiere al agua, es notable el grado de presión ejercido sobre las fuentes de abasto del vital líquido, haciendo que cada vez sea menos la capacidad de estas de abastecernos de acuerdo con nuestras necesidades y demanda, provocando su sobreexplotación.

A su vez, es visible la contaminación del agua generada por las industrias y la misma población. Esto ocasiona que las aguas superficiales $y$, por ende, los acuíferos no tengan las condiciones para abastecer, reduciendo la disponibilidad del líquido sin un correcto tratamiento. A causa de esto, se han indagado nuevas formas para el mantenimiento y aprovechamiento del mencionado líquido; a su vez han surgido conceptos que permiten analizar su ciclo.

Es por lo que, en el presente artículo, se analizan datos estadísticos nacionales referentes al agua para verificar la relación entre dos de sus variables: la precipitación y el agua renovable por entidad federativa. Esto, con el fin de corroborar el aprovechamiento del agua pluvial y la manera en que se refleja en la disponibilidad del agua en México.

Con base en lo anterior, surgen las preguntas de investigación que guiarán al mismo. La primera interroga si ¿La cantidad de precipitación pluvial está asociada a la cantidad de agua renovable? Aunado a esto, surge una segunda pregunta la cual plantea si ¿Existe un aprovechamiento del agua pluvial en relación con su aporte a la disponibilidad de agua potable en el país? Finalmente, con base en los resultados obtenidos, se plantean las posibles razones 
de falta de agua en el país a pesar de la gran cantidad de precipitación pluvial que este posee.

Se considera entonces, el planteamiento de una hipótesis basada en los conceptos antes mencionados la cual se refiere a que "si hay mayor precipitación, entonces aumenta la cantidad de agua renovable".

De manera que, a partir del coeficiente Gamma, se determinará si existe una relación de dependencia entre las variables mencionadas y así poder analizar los cuestionamientos antes planteados.

El objetivo principal del artículo es contrastar los resultados obtenidos a partir de la comparación dedosvariablesfundamentadas, con base en datos estadísticos nacionales, para verificar su relación y así determinar si en realidad el agua pluvial se aprovecha de manera adecuada y se refleja en el aumento de disponibilidad del agua renovable.

Antes de continuar, se menciona la importancia de determinar si el agua pluvial se aprovecha de una manera sustentable para contribuir al agua renovable, es decir, al agua disponible para diversos usos, la cual radica en que es necesario darle un correcto uso a dicha agua, y no desperdiciarla.

Finalmente, se hace indispensable analizar la causa de factores para que existan escasez del agua, los cuales se exponen a continuación, previo al análisis estadístico.

\section{El agua y su aprovechamiento.}

Para empezar, hay que considerar que el acceso al agua es un derecho humano (Asamblea General de las Naciones Unidas, 2010). Esta declaración nos hace conscientes de la vital importancia del líquido y su correcto uso. Por su parte, Marisa Mazari afirma que:

El agua cubre aproximadamente el 75\% de la superficie terrestre; es fundamental para los procesos tanto ambientales como sociales e indispensables para el surgimiento y desarrollo de la vida. En la actualidad estamos alterando los sistemas acuáticos a un ritmo acelerado y enfrentamos gravísimos problemas relacionados con el uso y mantenimiento de este valioso recurso. (p.10)

Si se analiza el agua como elemento, entonces podemos afirmar que se considera un recurso renovable debido a que presenta un ciclo hidrológico, por lo que mientras la lluvia no deje de caer, debe existir agua. Desgraciadamente, en la actualidad, las reservas de agua dulce se han utilizado más rápido de lo que tardan en recuperarse, por lo que el recurso comienza a transformarse en no renovable. Es por ello por lo que su aprovechamiento es fundamental para contribuir con su ciclo natural.

México tiene épocas del año con grandes cantidades de precipitación pluvial, esta cantidad depende de la región (Figura 1).

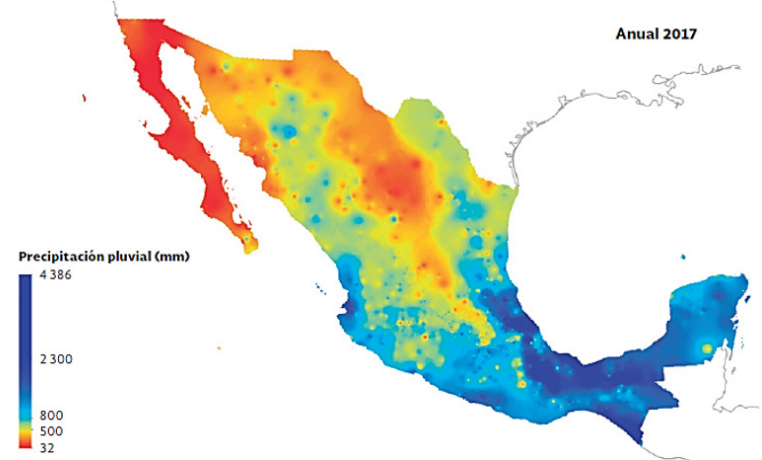

Figura 1. Precipitación Pluvial en México 2017. Fuente: elaborado con base en Conagua (2017).

Sin embargo, en México, a pesar de la cantidad de agua pluvial y su esperado ciclo, no se ve reflejado en el agua destinada a abastecer a la población (urbana y rural) ya que no ha logrado una correcta cobertura ni una óptima gestión. El abastecimiento del agua en México afronta diferentes problemáticas, como la mala gestión, explotación de los mantos acuíferos y pozos, así como el aumento de la necesidad del recurso derivado de la sobrepoblación y actividades económicas y agrarias.

El cuestionamiento en este sentido es: ¿Por qué a pesar de tener una alta precipitación pluvial en el país, también se presentan 
problemas de desabasto y escasez del recurso? La respuesta a esa pregunta va encaminada primero, al crecimiento demográfico y a la expansión de la mancha urbana, lo cual provoca que las ciudades cada día tengan menos espacios verdes de infiltración natural, ocasionando una ruptura tangente en el ciclo hídrico, lo que conduce a la no regeneración del recurso.

En segundo lugar, la infraestructura hidrosanitaria en México se basa en un sistema combinado, dicho sistema no separa las aguas pluviales de las aguas residuales, por lo que al final, las primeras se contaminan y desembocan en los drenajes urbanos, que no le dan ningún tipo de tratamiento y desembocan en cuerpos de agua superficiales, lo cual provoca la contaminación de estos. A su vez, existe la contaminación de suelos, ecosistemas, cuerpos de agua y agua subterránea (acuíferos), y esto hace que gran parte del agua potable que se logra recuperar, aún de manera natural, no sea adecuada para el consumo humano ni para la regeneración.

Por otro lado, la urbanización de las ciudades derivada de la industrialización y crecimiento demográfico en las mismas ha llegado al límite de poner en peligro las posibilidades de continuar disponiendo de agua, debido a que se rebasa la capacidad de renovación del recurso al necesitar cada vez más del mismo y no dando el tiempo necesario ni las condiciones para que se regenere (Rodríguez, 2014). Se estima que solamente el $0.77 \%$ del agua en el mundo es agua dulce accesible para consumo del ser humano.

En este sentido se infiere que, al explotar territorios para extracción de recursos, los mantos acuíferos y la tierra sufren impactos ambientales en grandes niveles. Las industrias invaden ecosistemas, los rompen y contaminan sus recursos propios. Los químicos utilizados en las industrias contaminan con sus deshechos a los ríos y mantos acuíferos cercanos y esto hace que el agua esté cada día más contaminada. Las industrias, usan grandes cantidades de agua que no está controlada ni regulada por los organismos responsables, haciendo que la población se quede sin recursos o que los recursos que tienen estén contaminados.

A partir de lo mencionado anteriormente, se rescata el concepto de agua renovable, el cual se entiende como la cantidad máxima de agua que resulta factible de explotar en una región sin alterar su ecosistema, es decir de manera sustentable. Esta agua se renueva y es capaz de cumplir un ciclo a partir de las aguas pluviales, de ahí que sea de suma importancia el aprovechamiento de estas.

De acuerdo con lo anterior y según el Atlas del Agua en México, de la cantidad anual de agua pluvial, se estima que el $72.1 \%$ se evapora, $21.4 \%$ escurre por ríos y arroyos y $6.4 \%$ se filtra al subsuelo naturalmente y recarga los acuíferos. Por tanto, se infiere que el $27.5 \%$ contribuye a la cantidad de agua renovable, esto tomando en cuenta los flujos de salida (exportaciones) y de entrada (importaciones) de agua con los países vecinos (CONAGUA, 2018).

Asimismo, el agua destinada al abastecimiento público y a la actividad económica o productiva, proviene en su mayoría de la precipitación pluvial. A esta agua es necesario, como se mencionó, sumarle el agua proveniente de los ríos que viajan por otros países y restarle el flujo que se evapora o sale por la vía fluvial. El agua disponible o renovable, entonces, es aquella que resulta de considerar estos aspectos y la cual se deposita en otros cuerpos de agua para infiltrarse en los acuíferos. A este proceso se le llama balance hídrico, el cual, a partir de lo ya analizado en el presente artículo, está siendo cada vez menos presente en el país.

De modo que, a continuación, se procede a determinar precisamente la relación que existe entre la precipitación pluvial y el agua renovable, para corroborar nuestra hipótesis y fundamentar una de las interrogantes que rigen la presente investigación. 


\section{METODOLOGÍA}

Para comenzar, y previo a examinar los resultados obtenidos, se describe el método estadístico utilizado para la obtención de estos con base en la siguiente metodología:

A partir de lo analizado acerca de la situación en México, se toman las variables ya mencionadas de precipitación pluvial y agua renovable (disponibilidad de agua) a partir de nuestra población referida a las entidades federativas de la República Mexicana.

Se determina que, para el análisis, la muestra es tomada a partir de los datos del 2018 correspondientes a la SEMARNAT y CONAGUA donde se encuentran las cifras más recientes de las variables.

A partir de la definición de las variables, se hace la aplicación del coeficiente Gamma (Y) o bien estadístico gamma, o gamma de Goodman y Kruskal el cual es un indicador de asociación. Las variables con las que este estadístico trabaja se encuentran en un nivel ordinal y para efectos del análisis dichas variables se distribuyen en cuatro criterios o intervalos respecto a la cantidad de agua: ALTA, MEDIA, BAJA Y MUY BAJA; para así poder realizar la tabla de contingencia $y$ realizar el cálculo.

Una vez obtenido el coeficiente, se define la relación de las variables ya sea positiva perfecta (1), correlación inversa perfecta $(-1)$ o bien cuando si no hay correlación entre las variables (0), para así deducir que cuanto más se acerque el coeficiente a 10 -1 , más fuerte es la correlación entre las mismas.

Posteriormente se procede a realizar la prueba de significación correspondiente y así poder comprobar la hipótesis planteada.

Finalmente, a partir de los cálculos y sus resultados, se presentan las conclusiones obtenidas para así corroborar nuestra hipótesis y responder a nuestras preguntas de investigación.

\section{RESULTADOS}

A continuación, se presentan los datos de las variables por entidad federativa en el 2018 , recabados por las fuentes oficiales y ordenadas de manera ascendente, a partir de los cuales se realiza el análisis (Tabla 1 ).

Tabla 1. Datos de las variables agua renovable y precipitación 2018.

\begin{tabular}{|c|c|c|c|}
\hline $\begin{array}{l}\text { ENTIDAD } \\
\text { FEDERATIVA }\end{array}$ & $\begin{array}{c}\text { Agua } \\
\text { renovable anual } \\
\text { (hm } 3)\end{array}$ & $\begin{array}{c}\text { ENTIDAD } \\
\text { FEDERATIVA }\end{array}$ & $\begin{array}{c}\text { Precipitación } \\
\text { pluvial anual } \\
\text { ( } \mathrm{mm})\end{array}$ \\
\hline Aguascalientes & 536 & Baja California Sur & 183 \\
\hline CDMx & 644 & Baja California & 199 \\
\hline Tlaxcala & 868 & Coahuila & 342 \\
\hline Baja California Sur & 1235 & Sonora & 427 \\
\hline Quintana Roo & 1741 & Chihuahua & 436 \\
\hline Morelos & 1848 & Aguascalientes & 477 \\
\hline Queretaro & 1940 & Durango & 499 \\
\hline Colima & 2159 & Zacatecas & 517 \\
\hline Baja California & 3045 & Queretaro & 571 \\
\hline Coahuila & 3457 & Nuevo León & 613 \\
\hline Guanajuato & 3886 & Guanajuato & 621 \\
\hline Zacatecas & 4016 & CDMx & 714 \\
\hline Nuevo León & 4448 & Tlaxcala & 715 \\
\hline Mexico & 4786 & Sinaloa & 768 \\
\hline Campeche & 5815 & Tamaulipas & 777 \\
\hline Nayarit & 6660 & Hidalgo & 792 \\
\hline Sonora & 7154 & Michoacan & 819 \\
\hline Hidalgo & 7475 & Jalisco & 833 \\
\hline Tamaulipas & 8962 & Mexico & 866 \\
\hline Sinaloa & 9903 & San Luis Potosí & 941 \\
\hline San Luis Potosí & 10862 & Morelos & 955 \\
\hline Puebla & 11382 & Colima & 966 \\
\hline Chihuahua & 11759 & Yucatán & 1077 \\
\hline Durango & 12594 & Nayarit & 1102 \\
\hline Michoacan & 12633 & Guerrero & 1116 \\
\hline Jalisco & 15951 & Campeche & 1201 \\
\hline Guerrero & 20972 & Quintana Roo & 1276 \\
\hline Yucatán & 21813 & Puebla & 1277 \\
\hline Tabasco & 31941 & Oaxaca & 1486 \\
\hline Veracruz & 51640 & Veracruz & 1511 \\
\hline Oaxaca & 55901 & Chiapas & 1991 \\
\hline Chiapas & 113557 & Tabasco & 2390 \\
\hline \multicolumn{2}{|c|}{ ALTA 14001 o más } & ALTA & 1101 o más \\
\hline MEDIA & $9001-14000$ & MEDIA & $801-1100$ \\
\hline BAIA & $4001-9000$ & BAUA & $501-800$ \\
\hline MUY BAVA & 4000 o menos & MUY BAUA & 500 o menos \\
\hline
\end{tabular}

Fuente: Elaboración propia con base en SEMARNAT Y CONAGUA (2018)

Es así como, a partir de los datos antes presentados, se realiza el cálculo estadístico donde se obtiene lo siguiente:

El coeficiente Gamma $=0.54$, el cual describe una asociación positiva, es decir, existe una fuerte relación entre el agua renovable y la precipitación pluvial en los estados de la 
República. Por su parte, en el resultado de la prueba de significación, la $Z$ calculada $=$ 3.61 , lo que demuestra que las variables analizadas son dependientes entre sí.

Asimismo, analizando la tabla de contingencia realizada para el cálculo mostrada a continuación, se observa que hay gran cantidad de estados que presentan grandes precipitaciones y se ve reflejado en la cantidad de agua renovable. Pero existen estados que presentan cantidades muy bajas de precipitación y cantidades medias o altas de agua renovable, por lo que se consideraría interesante investigar la fuente de generación de las mismas (Tabla 2).

Tabla 2. Tabla de contingencia

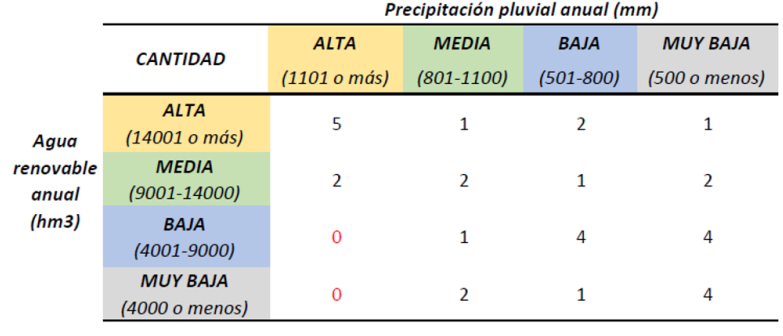

Fuente: Elaboración propia con base en SEMARNAT Y CONAGUA (2018).

Nota: La tabla muestra la cantidad de estados de la República Mexicana con las relaciones de los intervalos correspondientes de ambas variables.

\section{DISCUSIÓN}

A partir del cálculo realizado, se obtiene el resultado de que efectivamente la cantidad de agua pluvial y de agua renovable tienen una fuerte relación positiva. Se infiere que, el agua pluvial contribuye de una manera significativa a la disponibilidad de agua del país. Surge entonces el cuestionamiento presentado anteriormente: ¿Si México tiene un alto índice de precipitación pluvial anual, a qué se debe la escasez del agua potable?

Acorde con las cifras internacionales, a pesar de la cantidad de precipitación, se afirma que México tiene una disponibilidad de $0.1 \%$ del total del agua dulce del planeta, siendo su distribución bastante desigual. En la clasificación mundial, México está considerado como un país con baja disponibilidad (Maguey, 2018). Derivado de estos datos, se infiere que el agua pluvial, no está aportando lo supuesto para la correcta regeneración del recurso.

Como ya se mencionó anteriormente, y rectificando los fundamentos que soportan la presente investigación; se afirma que, el crecimiento poblacional e industrial; la creciente falta de espacios verdes para infiltración natural del mismo; la falta de estrategias de gestión y aprovechamiento pluvial; y la contaminación de los cuerpos de agua, ocasionan múltiples problemáticas de las cuales se obtienen las siguientes afirmaciones:

El aumento de la necesidad de recursos hídricos para la población y sector económico se hace cada vez mayor, por lo que existe una sobreexplotación del recurso al no dar tiempo de regeneración de este y continuar disponiendo de él de una manera descontrolada.

Existe una imposibilidad de infiltración de aguas pluviales dentro de las urbes, por lo que el agua pluvial no cumple su objetivo de renovar naturalmente el recurso quedando, a su vez, desaprovechada.

Las ciudades industrializadas no tienen un control del gasto hídrico del sector, al permitir que pueda hacer uso desmedido del agua sin políticas que lo regulen y a su vez que derivado de sus desechos, contaminen los cuerpos de agua cercanos.

Los factores antes mencionados provocan que el agua renovable no sea suficiente para abastecer la totalidad del recurso demandado por el país provocando una baja disponibilidad del recurso y contaminando las fuentes que pueden contribuir con la renovación.

Estas problemáticas se presentan de manera internacional desde hace ya varios años. A partir de esto, surgen decretos, leyes, organismos y propuestas nacionales e internacionales que destacan la urgente necesidad del implemento de estrategias encaminadas a el aprovechamiento pluvial y a la regeneración y mantenimiento hídrico por ejemplo en materia internacional la 
Agenda 2030 y la Organización de las Naciones Unidad; nacionalmente surgen leyes, normas y planes hídricos, planes de desarrollo urbano, entre otros. De manera lamentable, no han sido correctamente implementados y no existen políticas públicas adecuadas para la implementación de dichas estrategias por lo que la problemática en el país va en aumento.

Los organismos se han ocupado más en el manejo y distribución de agua potable que en la preservación de cuerpos hídricos. Para poder realizar un manejo sustentable del agua hay que considerar algunos aspectos. Primero, la valorización de costos y tarifas por región que no podemos dejar de lado; segundo, las prácticas del manejo en relación con su uso que permitan la conservación, recolección y aprovechamiento del agua; y por último, sistemas de manejo urbano del agua y de saneamiento ambiental que se encaminen a políticas y estrategias sustentables para el aprovechamiento y gestión de las aguas pluviales.

Se ha determinado el agua como un bien común o público, lo cual crea crisis ya que debido a la falta infraestructura no llega a todos los asentamientos, por otra parte ocasiona que la población que si tiene agua constantemente, no tenga una conciencia de ahorro de la misma (Leff, 2008).

Por último, es necesario resaltar la sustentabilidad como parte de las estrategias de las metrópolis enfatizando la importancia de orientar la competitividad y crecimiento económico, al mismo tiempo que se mejore la calidad de vida conservando los recursos naturales y teniendo un respeto a la cultura e identidad (Cabrera, 2010).

\section{CONCLUSIONES}

Para concluir $y$, una vez realizado el procedimiento estadístico que nos permitió la comprobación de nuestra hipótesis, podemos afirmar que, estadísticamente, la cantidad de precipitación pluvial si está asociada a la cantidad de agua renovable de una forma determinante, es decir, mientras más agua pluvial exista debería de existir una mayor disponibilidad de esta.

En el caso de México, las cifras nos arrojan un alta precipitación pluvial pero una baja disponibilidad, por lo que, teniendo en cuenta la prueba estadística y la hipótesis comprobada; se determina que a pesar de que hay una alta cantidad de lluvias en el país, no se están aprovechando para regenerar el recurso y así contribuir con el agua renovable; es decir, el agua pluvial no se está infiltrando de manera natural para la recarga de acuíferos y por el contrario, como se analizó en el presente artículo, está siendo contaminada. Por lo que en relación con la segunda pregunta planteada, se concluye que no existe un suficiente aprovechamiento del agua pluvial en relación con su aporte a la disponibilidad de agua potable en el país.

Del mismo modo, y derivado de lo anterior, se afirma que, el agua renovable no está siendo suficiente para abastecer a las necesidades actuales de agua potable de la población del país.

Más aún, se destaca la importancia de poder tener un aprovechamiento visible de agua. Si bien en México aún no se implementan políticas públicas con estrategias obligatorias para la recuperación y tratamiento del agua pluvial, se hace necesario que ya se haga para así crear las condiciones adecuadas para que esta se logre infiltrar con la menor cantidad de pérdidas y contaminantes posibles. Asimismo, dichas políticas públicas deben abarcar la problemática de regulación del gasto y el aprovechamiento del recurso hídrico.

Acorde con lo mencionado, es indispensable poder reducir la demanda incrementando la eficiencia de los sistemas de distribución de agua en las ciudades y de los sistemas de riego en el sector agrícola; e incrementar la oferta aumentando el volumen de agua pluvial recolectada para su reúso.

En definitiva, todos los ámbitos de la sociedad (gobierno, población, empresas, escuelas, etcétera) debemos unirnos para crear una sociedad global sustentable, la cual 
se defina por aspectos económicos, sociales y ambientales analizados con la misma importancia unos de otros. Es la sociedad la que determina el rumo del planeta por lo que es necesario preservar la biósfera ya que de ella obtenemos los recursos necesarios para la vida.

Por su parte, el consumismo, la globalización, la industrialización y el calentamiento global se han encargado de devastar a la tierra, nos agotamos los recursos y extinguimos especies. El aumento de la población ha sobrecargado los sistemas ecológicos y sociales. Es necesario adquirir responsabilidad universal, una visión de los valores básicos parea así constituir sociedades democráticas que preserven la tierra con integridad ecológica, evitando dañar el ambiente, y teniendo una educación sustentable.

Finalmente se destaca que, la implementación de principios e ideas sustentables en relación con el agua como el aprovechamiento y tratamiento de aguas pluviales, la preservación de mantos acuíferos, y el ahorro y cuidado de la misma, suman mucho a la difícil tarea de la humanidad y su creciente deuda con el ambiente y el planeta.

\section{REFERENCIAS}

Boisier, S. (2001). El territorio en la globalización. Notas del expositor para una exposición.

Cabrera, V. (2010). Diagnóstico de competitividad en el municipio de Puebla. México: Benemérita Universidad Autónoma de Puebla.

Cienfuegos-Velasco, M. Á., Aguilar, M., \& Alejo-Pompilio, A. (2012). Política descentralizadora del agua potable. Espacios Públicos, vol. 15, núm. 35, septiembre-diciembre, PP.85-95.

CONAGUA. (2018). Estadísticas del agua en México. México: Secretaría de Medio Ambiente y Recursos Naturales. Recuperado de: https://apps1.semarnat.gob.mx:8443/dgeia/informe18/tema/cap6.html

Gudynas, E. (2001). Extractivismos. Ecología, economía y política de un modo de entender el desarrollo y la Naturaleza, primera edición, 2015: CEDIB.

Leff, E. (2008). Discursos Sustentables. México: Siglo XXI Editores.

Maguey, H. (2018). Más de $80 \%$ del agua se va en uso agrícola y de la industria. Gaceta UNAM. Recuperado de: https://www.gaceta.unam.mx/crisis-agua-industria/

Mazari, M. (s/f). El agua como recurso. ¿Cómo ves? Revista de divulgación de ciencia de la UNAM. PP. 10-12.

PNUMA. (2007). Perspectivas del Medio Ambiente Mundial. En PNUMA, Medio Ambiente para el desarrollo. Dinamarca: GEO4.

RodríguezPeñaloza, Martín, et., al. (2014). Desarrollosostenibleydesarrollosustentable: unanálisis epistemológico diferenciad. 1er. Congreso Internacional Desarrollo Sustentable: Enfoques, Aplicaciones y Perspectivas "Ambiente, economía, sociedad, territorio y educación" CEDESUAEMéx., del 5 al 7 de noviembre, 2014. Centro Cultural Universitario, Toluca, México. PP. 1-19.

Secretaría de Medio Ambiente y Recursos Naturales. (2020). Informe del Medio Ambiente. México: Sistema Nacional de Información Ambiental y Recursos Naturales. 